\title{
THE CHECKERBOARD FAMILY OF ENTANGLED STATES OF TWO QUTRITS
}

\author{
DRAGOMIR Ž.ĐOKOVIĆ
}

\begin{abstract}
By modifying the method of Bruß and Peres, we construct two new families of entangled two qutrit states. For all density matrices $\rho$ in these families we have $\rho_{i j}=0$ for $i+j$ odd. The first family depends on 27 independent real parameters and includes both PPT and NPT states. The second family consists of PPT entangled states. The number of independent real parameters of this family is $\geq 11$.
\end{abstract}

\section{INTRODUCTION}

Let $\mathcal{H}=\mathcal{H}_{A} \otimes \mathcal{H}_{B}$ be the Hilbert space for the quantum system consisting of two parties, A and B. A product state is a tensor product $\rho_{A} \otimes \rho_{B}$ of the states $\rho_{A}$ and $\rho_{B}$ of the first and second party, respectively. A state $\rho$ is separable if it can be written as a convex linear combination of product states. We say that a state $\rho$ is entangled if $\rho$ is not separable. It is PPT if its partial transpose $\rho^{\Gamma}=(1 \otimes T)(\rho)$ is a positive semidefinite operator. Otherwise it is NPT.

The Peres-Horodecki criterion [14, 9] settles the separability problem for $2 \otimes 2$ and $2 \otimes 3$ bipartite quantum systems, but in general it provides only a necessary condition for separability. Among bipartite systems, the $3 \otimes 3$ case plays a special role since it is the smallest case for which this criterion of separability is not sufficient. There are many papers, such as 1, 2, 4, 6, 7, 12, 11, 13, where entangled states of two qutrits or families of such states with special properties have been constructed and explored.

In their short note [4] Bruß and Peres constructed two families of PPT entangled states for two qutrits. Their first family depends on 5 independent real parameters and consists of states which are fixed under partial transposition, with respect to the chosen orthonormal basis of $\mathcal{H}_{B}$. (We do not consider at all their second family.) We shall extend this family to a much richer family, $\mathcal{F}^{\prime}$, which depends on at least 11 independent real parameters (see Theorem 4.1 and the subsequent remark). The states $\rho$ in our family are also PPT entangled and fixed under the partial transposition.

We begin with the four nonzero complex vectors

$$
\begin{aligned}
& \left|v_{1}\right\rangle=|a, 0, b ; 0, c, 0 ; d, 0, e\rangle, \\
& \left|v_{2}\right\rangle=|0, f, 0 ; g, 0, h ; 0, i, 0\rangle, \\
& \left|v_{3}\right\rangle=|j, 0, k ; 0, l, 0 ; m, 0, n\rangle, \\
& \left|v_{4}\right\rangle=|0, p, 0 ; q, 0, r ; 0, s, 0\rangle,
\end{aligned}
$$

where the components are listed in the order $00,10,20 ; 01, \ldots$. Note that $\left|v_{1}\right\rangle$ and $\left|v_{3}\right\rangle$ are orthogonal to $\left|v_{2}\right\rangle$ and $\left|v_{4}\right\rangle$, but $\left|v_{1}\right\rangle$ and $\left|v_{3}\right\rangle$, and also $\left|v_{2}\right\rangle$ and $\left|v_{4}\right\rangle$, in general are not orthogonal to each other. For comparison note that the four vectors 
used in 44 are pairwise orthogonal by construction. By using the above vectors, we construct the normalized state $\rho$, i.e., with $\operatorname{tr}(\rho)=1$,

$$
\rho=\frac{1}{N} \sum_{j=1}^{4}\left|v_{j}\right\rangle\left\langle v_{j}\right|, \quad N=\sum_{j=1}^{4}\left\langle v_{j} \mid v_{j}\right\rangle .
$$

In the next section we prove that this $\rho$ provides a family, $\mathcal{F}$, of entangled states of two qutrits. As we show later, $\mathcal{F}$ depends on 27 independent real parameters. We refer to it as the checkerboard family since the corresponding density matrices $\rho$ have the "checkerboard pattern", i.e., $\rho_{i j}=0$ for $i+j$ odd.

In section 4 we construct a subfamily $\mathcal{F}^{\prime} \subset \mathcal{F}$ consisting of states $\rho$ which are fixed under partial transposition. Thus, we obtain a new family of PPT entangled states of two qutrits. We will show later that $\mathcal{F}^{\prime}$ depends on at least 11 independent real parameters. It contains generically the first Bruß-Peres family. In section 5 we prove the two claims on the number of independent real parameters. In the last section we summarize and discuss our results.

\section{Proving that $\rho$ is Entangled}

As in 4, we use the range criterion of P. Horodecki [12] to prove that $\rho$ is entangled in the generic case. It suffices to show that the subspace spanned by the $\left|v_{j}\right\rangle$ contains no nonzero tensor product of two vectors.

Assume that

$$
|\alpha, \beta, \gamma\rangle \otimes|\delta, \varepsilon, \zeta\rangle=\sum A_{j}\left|v_{j}\right\rangle \neq 0
$$

This equation is equivalent to the system of two matrix equations:

$$
\begin{aligned}
& {\left[\begin{array}{ll}
a & j \\
b & k \\
c & l \\
d & m \\
e & n
\end{array}\right] \cdot\left[\begin{array}{l}
A_{1} \\
A_{3}
\end{array}\right]=\left[\begin{array}{l}
\alpha \delta \\
\gamma \delta \\
\beta \varepsilon \\
\alpha \zeta \\
\gamma \zeta
\end{array}\right],} \\
& {\left[\begin{array}{ll}
f & p \\
g & q \\
h & r \\
i & s
\end{array}\right] \cdot\left[\begin{array}{l}
A_{2} \\
A_{4}
\end{array}\right]=\left[\begin{array}{l}
\beta \delta \\
\alpha \varepsilon \\
\gamma \varepsilon \\
\beta \zeta
\end{array}\right] .}
\end{aligned}
$$

Since $(\alpha \delta) \cdot(\gamma \zeta)=(\gamma \delta) \cdot(\alpha \zeta)$, Eq. (2.1) implies that

$$
\left(a A_{1}+j A_{3}\right)\left(e A_{1}+n A_{3}\right)=\left(b A_{1}+k A_{3}\right)\left(d A_{1}+m A_{3}\right),
$$

i.e.,

$$
F\left(A_{1}, A_{3}\right):=(a e-b d) A_{1}^{2}+(a n+e j-b m-d k) A_{1} A_{3}+(j n-k m) A_{3}^{2}=0 .
$$

Similarly, from Eqs. (2.1) and (2.2) we obtain that

$$
\begin{aligned}
\left(f A_{2}+p A_{4}\right)\left(g A_{2}+q A_{4}\right) & =\left(a A_{1}+j A_{3}\right)\left(c A_{1}+l A_{3}\right), \\
\left(h A_{2}+r A_{4}\right)\left(i A_{2}+s A_{4}\right) & =\left(e A_{1}+n A_{3}\right)\left(c A_{1}+l A_{3}\right), \\
\left(g A_{2}+q A_{4}\right)\left(i A_{2}+s A_{4}\right) & =\left(d A_{1}+m A_{3}\right)\left(c A_{1}+l A_{3}\right), \\
\left(f A_{2}+p A_{4}\right)\left(h A_{2}+r A_{4}\right) & =\left(b A_{1}+k A_{3}\right)\left(c A_{1}+l A_{3}\right) .
\end{aligned}
$$


These four equations can be written in matrix form

$$
\left[\begin{array}{cccc}
f g & f q+g p & p q & \left(a A_{1}+j A_{3}\right)\left(c A_{1}+l A_{3}\right) \\
h i & h s+i r & r s & \left(e A_{1}+n A_{3}\right)\left(c A_{1}+l A_{3}\right) \\
f h & f r+h p & p r & \left(d A_{1}+m A_{3}\right)\left(c A_{1}+l A_{3}\right) \\
g i & g s+i q & q s & \left(b A_{1}+k A_{3}\right)\left(c A_{1}+l A_{3}\right)
\end{array}\right] \cdot\left[\begin{array}{c}
A_{2}^{2} \\
A_{2} A_{4} \\
A_{4}^{2} \\
-1
\end{array}\right]=0 .
$$

Hence this $4 \times 4$ matrix must be singular, which gives

$$
(f s-i p)(g r-h q)\left(c A_{1}+l A_{3}\right)\left(\lambda A_{1}+\mu A_{3}\right)=0,
$$

where

$$
\begin{aligned}
\lambda & =a(h s-i r)+b(i q-g s)+d(f r-h p)+e(g p-f q), \\
\mu & =f(m r-n q)+g(n p-k s)+h(j s-m p)+i(k q-j r) .
\end{aligned}
$$

We now assume that

$$
(f s-i p)(g r-h q) F(l,-c) F(\mu,-\lambda) \neq 0 .
$$

Then $c A_{1}+l A_{3}=0$ or $\lambda A_{1}+\mu A_{3}=0$. Because $F(l,-c) \cdot F(\mu,-\lambda) \neq 0$, neither of these two linear equations has a common nontrivial solution with Eq. (2.3). Consequently, we must have $A_{1}=A_{3}=0$. The four equations below Eq. (2.3) now imply that $A_{2}=A_{4}=0$, which is a contradiction.

Thus the following theorem holds.

Theorem 2.1. Let $a, b, \ldots, n, p, q, r, s$ be 18 complex numbers subject to the condition (2.6), where $\lambda$ and $\mu$ are defined by the Eqs. 2.4) and (2.5), and $F$ by Eq. (2.3). Then the state $\rho$ given by (1.1) is entangled.

We denote by $\mathcal{F}$ the family of entangled states $\rho$ provided by this theorem. We shall see later that $\mathcal{F}$ depends only on 27 independent real parameters.

It is easy to test whether a given state $\rho \in \mathcal{F}$ is PPT. One just has to compute $\rho^{\Gamma}$ and apply to it the well known criterion for a hermitian matrix to be positive semidefinite. An interesting open question is to decide whether there is a state $\rho \in \mathcal{F}$ such that $\rho^{\Gamma}$ is positive definite.

\section{TwO EXAMPLES}

Note that while each $\rho \in \mathcal{F}$ has rank at most 4 , its partial transpose $\rho^{\Gamma}$ may be nonsingular. We give here two examples of distillable states $\rho \in \mathcal{F}$ which are of different types. In both examples $\rho^{\Gamma}$ is nonsingular.

The reduction criterion provides also a necessary condition for separability of bipartite states $\rho$. It requires that the matrices $\rho_{A} \otimes 1-\rho$ and $1 \otimes \rho_{B}-\rho$ be positive semidefinite, where $\rho_{A}$ and $\rho_{B}$ are the two reduced density matrices of $\rho$. If $\rho$ violates the reduction criterion then it is entangled. Furthermore, in that case $\rho$ is also distillable [8].

Our first example $\rho_{1}$ violates the reduction criterion and so it is distillable. The components $a, b, \ldots, s$ are chosen as follows: $a=f=k=n=q=s=0$, $g=p=m=1, j=l=-1, h=i=\mathbf{i}, e=1-\mathbf{i}, b=d=-1+\mathbf{i}, c=r=-1-\mathbf{i}$, where $\mathbf{i}$ is the imaginary unit. A computation shows that $\operatorname{det}\left(\rho_{1}^{\Gamma}\right)=2^{6} \cdot 7 / 17^{9}$ and that $\rho_{1}^{\Gamma}$ has exactly two negative eigenvalues. We recall from [10] that all distillable 
states are NPT. The density matrix is

$$
\rho_{1}=\frac{1}{17}\left[\begin{array}{ccccccccc}
1 & 0 & -1 & 0 & 1 & 0 & 0 & 0 & 0 \\
0 & 1 & 0 & 0 & 0 & -\mathbf{i} & 0 & -\mathbf{i} & 0 \\
-1 & 0 & 3 & 0 & -1-2 \mathbf{i} & 0 & 2 & 0 & -2 \\
0 & 0 & 0 & 1 & 0 & 0 & 0 & -1+\mathbf{i} & 0 \\
1 & 0 & -1+2 \mathbf{i} & 0 & 3 & 0 & 2 \mathbf{i} & 0 & -2 \mathbf{i} \\
0 & \mathbf{i} & 0 & 0 & 0 & 1 & 0 & 1 & 0 \\
0 & 0 & 2 & 0 & -2 \mathbf{i} & 0 & 2 & 0 & -2 \\
0 & \mathbf{i} & 0 & -1-\mathbf{i} & 0 & 1 & 0 & 3 & 0 \\
0 & 0 & -2 & 0 & 2 \mathbf{i} & 0 & -2 & 0 & 2
\end{array}\right] .
$$

Our second example is a state $\rho_{2} \in \mathcal{F}$ which is distillable but satisfies the reduction criterion: $a=b=c=f=j=m=p=r=1, n=0, e=-1, q=s=\mathbf{i}$, $g=h=-\mathbf{i}, d=1+\mathbf{i}, i=-1-\mathbf{i}, k=l=-1+\mathbf{i}$. The density matrix is

$$
\rho_{2}=\frac{1}{21}\left[\begin{array}{ccccccccc}
2 & 0 & 2-\mathbf{i} & 0 & -\mathbf{i} & 0 & -\mathbf{i} & 0 & -1 \\
0 & 2 & 0 & 0 & 0 & 2+\mathbf{i} & 0 & 1+\mathbf{i} & 0 \\
2+\mathbf{i} & 0 & 3 & 0 & 0 & 0 & 0 & 0 & -1-\mathbf{i} \\
0 & 0 & 0 & 2 & 0 & -1 & 0 & 1+\mathbf{i} & 0 \\
\mathbf{i} & 0 & 0 & 0 & 3 & 0 & 3 & 0 & -1 \\
0 & 2-\mathbf{i} & 0 & -1 & 0 & 3 & 0 & 1 & 0 \\
\mathbf{i} & 0 & 0 & 0 & 3 & 0 & 3 & 0 & -1 \\
0 & 1-\mathbf{i} & 0 & 1-\mathbf{i} & 0 & 1 & 0 & 2 & 0 \\
-1 & 0 & -1+\mathbf{i} & 0 & -1 & 0 & -1 & 0 & 1
\end{array}\right] .
$$

The vector $|\psi\rangle=\left|\varphi_{1}\right\rangle \otimes\left|\varphi_{2}\right\rangle+\left|\varphi_{3}\right\rangle \otimes\left|\varphi_{4}\right\rangle$, where

$$
\left|\varphi_{1}\right\rangle=\left[\begin{array}{c}
-\mathbf{i} \\
1-\mathbf{i} \\
1-\mathbf{i}
\end{array}\right], \quad\left|\varphi_{2}\right\rangle=\left[\begin{array}{c}
1-\mathbf{i} \\
-1+\mathbf{i} \\
1
\end{array}\right], \quad\left|\varphi_{3}\right\rangle=\left[\begin{array}{c}
1-\mathbf{i} \\
\mathbf{i} \\
0
\end{array}\right], \quad\left|\varphi_{4}\right\rangle=\left[\begin{array}{c}
-1 \\
-\mathbf{i} \\
1
\end{array}\right],
$$

has Schmidt rank 2, and a computation shows that $\left\langle\psi\left|\rho_{2}^{\Gamma}\right| \psi\right\rangle=-5 / 21$ is negative. Therefore $\rho_{2}$ is 1 -distillable. We have $\operatorname{det}\left(\rho_{2}^{\Gamma}\right)=2 \cdot 11 \cdot 19 /\left(3^{7} 7^{9}\right)$ and exactly two eigenvalues of $\rho_{2}^{\Gamma}$ are negative.

\section{Forcing $\rho$ to Be FiXed By the Partial transposition}

Recall that all states $\rho$ of the first Bruß-Peres family are not only PPT but also satisfy $\rho^{\Gamma}=\rho$. We shall also impose this stronger condition.

In this section we assume that $\rho \in \mathcal{F}$ and that the conditions of Theorem 2.1 hold. The equality $\rho^{\Gamma}=\rho$ holds if and only if the following eight conditions are satisfied. The first five conditions are

$$
\begin{aligned}
f g^{*}+p q^{*} & =c a^{*}+l j^{*}, \\
i g^{*}+s q^{*} & =c d^{*}+l m^{*}, \\
f h^{*}+p r^{*} & =c b^{*}+l k^{*}, \\
i h^{*}+s r^{*} & =c e^{*}+l n^{*}, \\
a e^{*}+j n^{*} & =d b^{*}+m k^{*},
\end{aligned}
$$

and the remaining three are

$$
\Im\left(a d^{*}+j m^{*}\right)=\Im\left(b e^{*}+k n^{*}\right)=\Im\left(f i^{*}+p s^{*}\right)=0,
$$


where $\Im(z)$ denotes the imaginary part of the complex number $z$. In the following analysis we shall assume that all denominators in our formulae are nonzero.

Since $f s-i p \neq 0$, the equations (4.1) and (4.2) can be solved for $g$ and $q$, and the equations (4.3) and (4.4) for $h$ and $r$ :

$$
\begin{aligned}
g & =\frac{s^{*}\left(a c^{*}+j l^{*}\right)-p^{*}\left(d c^{*}+m l^{*}\right)}{(f s-i p)^{*}}, \\
q & =\frac{f^{*}\left(d c^{*}+m l^{*}\right)-i^{*}\left(a c^{*}+j l^{*}\right)}{(f s-i p)^{*}}, \\
h & =\frac{s^{*}\left(b c^{*}+k l^{*}\right)-p^{*}\left(e c^{*}+n l^{*}\right)}{(f s-i p)^{*}}, \\
r & =\frac{f^{*}\left(e c^{*}+n l^{*}\right)-i^{*}\left(b c^{*}+k l^{*}\right)}{(f s-i p)^{*}} .
\end{aligned}
$$

From Eqs. (4.6) we obtain that

$$
d=\left(x-m j^{*}\right) / a^{*}, \quad e=\left(y-n k^{*}\right) / b^{*}, \quad i=\left(t-s p^{*}\right) / f^{*},
$$

where $x, y$, and $t$ are real numbers. By plugging in the above expressions for $d$ and $e$ into Eq. (4.5) and solving it for $n$, we obtain that

$$
n=\frac{|a|^{2} y-|b|^{2} x-m^{*} b^{*}(a k-b j)}{a(a k-b j)^{*}} .
$$

To summarize, we have the following result.

Theorem 4.1. Let $t, x, y$ be real and $a, b, c, f, j, k, l, m, p, s$ complex parameters and define $g, q, h, r, d, e, i, n$ by the formulae (4.7-12). Assume that

$$
a b f(f s-i p)(g r-h q)(a k-b j) F(l,-c) F(\mu,-\lambda) \neq 0,
$$

where $\lambda$ and $\mu$ are defined by Eqs. 2.4) and (2.5) and F by Eq. (2.3). Then the state $\rho$ given by (1.1) is entangled and satisfies $\rho^{\Gamma}=\rho$. In particular, $\rho$ is a PPT state.

We denote by $\mathcal{F}^{\prime}$ the subfamily of $\mathcal{F}$ singled out by this theorem. Since all states $\rho \in \mathcal{F}^{\prime}$ satisfy $\rho^{\Gamma}=\rho$, it is clear that $\mathcal{F}^{\prime}$ does not contain the second Bruß-Peres family.

On the other hand, we remark that $\mathcal{F}^{\prime}$ contains generically their first family. To prove this we set

$$
k=y=0, j=c^{*}, l=-a^{*}, m=x / c, p=t c f^{*} / x a, s=x a^{*} / f c^{*} .
$$

Then the above formulae for $g, q, h, r, d, e, i, n$ give

$$
d=e=i=n=r=0, g=t f c^{*}, q=-f^{*}, h=b c^{*} / f^{*},
$$

and from the Eq. (2.3) we obtain that

$$
F(l,-c)=-x b a^{*}, \quad F(\mu,-\lambda)=\frac{x\left(b c^{*}\right)^{3}}{\left|a c f^{2}\right|^{2}} \cdot\left(x|a|^{2}-t|f|^{2}\right)^{2} .
$$

By assuming that also $a, b, c, f$ are real and that $a b c f x\left(x|a|^{2}-t|f|^{2}\right) \neq 0$, we obtain generically the first Bruß-Peres family. Explicitly, our parameters $a, b, c, f, t, x$ correspond to $m, a c / n, n, a, b / a n, a d n / m$, respectively, in the Bruß-Peres family. If we allow $a, b, c, f$ to remain complex, the number of independent real parameters increases from 5 to 7 . 


\section{Counting Independent REAL PARAMETERS}

We consider first the family $\mathcal{F}$ provided by Theorem 2.1. The state $\rho$ depends on 36 real parameters (the real and imaginary parts of the 18 complex parameters). However, we claim that there are only 27 independent such parameters. In order to prove this claim, it is convenient to drop the normalization factor $1 / N$ and show that the modified family $\Phi:(a, b, \ldots, s) \mapsto N \rho$ depends on 28 independent parameters.

Since the rank of $d \Phi$ can take only finitely many values, there exists a point $\omega_{0}=\left(a_{0}, b_{0}, \ldots, s_{0}\right)$ in the domain of $\Phi$ at which the rank of $d \Phi$ takes the maximal value, say $\delta$. By continuity of $d \Phi$, its rank has to be $\delta$ in some small neighborhood $U$ of the point $\omega_{0}$. Now the Rank Theorem [3, Theorem 7.1] implies that the image $\Phi(U) \subseteq \mathcal{F}$ is a manifold of dimension $\delta$. Therefore the number of independent real parameters of $\mathcal{F}$ is at least $\delta$.

We shall first find an upper bound for $\delta$. Due to the checkerboard pattern of $\rho$, by simultaneous permutation of rows and columns of the matrix $N \rho$ we obtain the direct sum $\rho^{\prime} \oplus \rho^{\prime \prime}$ where

$$
\rho^{\prime}=\left[\begin{array}{cccc}
g g^{*}+q q^{*} & g f^{*}+q p^{*} & g i^{*}+q s^{*} & g h^{*}+q r^{*} \\
f g^{*}+p q^{*} & f f^{*}+p p^{*} & f i^{*}+p s^{*} & f h^{*}+p r^{*} \\
i g^{*}+s q^{*} & i f^{*}+s p^{*} & i i^{*}+s s^{*} & i h^{*}+s r^{*} \\
h g^{*}+r q^{*} & h f^{*}+r p^{*} & h i^{*}+r s^{*} & h h^{*}+r r^{*}
\end{array}\right]
$$

and

$$
\rho^{\prime \prime}=\left[\begin{array}{ccccc}
a a^{*}+j j^{*} & a d^{*}+j m^{*} & a c^{*}+j l^{*} & a b^{*}+j k^{*} & a e^{*}+j n^{*} \\
d a^{*}+m j^{*} & d d^{*}+m m^{*} & d c^{*}+m l^{*} & d b^{*}+m k^{*} & d e^{*}+m n^{*} \\
c a^{*}+l j^{*} & c d^{*}+l m^{*} & c c^{*}+l l^{*} & c b^{*}+l k^{*} & c e^{*}+l n^{*} \\
b a^{*}+k j^{*} & b d^{*}+k m^{*} & b c^{*}+k l^{*} & b b^{*}+k k^{*} & b e^{*}+k n^{*} \\
e a^{*}+n j^{*} & e d^{*}+n m^{*} & e c^{*}+n l^{*} & e b^{*}+n k^{*} & e e^{*}+n n^{*}
\end{array}\right] .
$$

Both $\rho^{\prime}$ and $\rho^{\prime \prime}$ have rank 2. For instance, the nullspace of $\rho^{\prime}$ is spanned by the columns of the matrix

$$
\left[\begin{array}{cc}
(f s-i p)^{*} & (f r-h p)^{*} \\
(i q-s g)^{*} & (h q-g r)^{*} \\
(g p-f q)^{*} & 0 \\
0 & (g p-f q)^{*}
\end{array}\right]
$$

It follows that the rank of $d \Phi$ is the same as that of the map

$$
\Psi:(a, b, \ldots, s) \mapsto \sigma^{\prime} \oplus \sigma^{\prime \prime},
$$

where $\sigma^{\prime}$ resp. $\sigma^{\prime \prime}$ is the submatrix of $\rho^{\prime}$ resp. $\rho^{\prime \prime}$ consisting of the first two columns. Since $\rho^{\prime}$ and $\rho^{\prime \prime}$ are hermitian, the image of $\Psi$ is contained in a 28-dimensional real vector space. Consequently, $\Psi$ depends on at most 28 independent real parameters and $\delta \leq 28$.

On the other hand, a computation shows that the rank of $d \Psi$ at the point used to define the state $\rho_{2}$ (see Sect. 3) is indeed 28. We conclude that $\delta=28$ and our claim follows.

Next we consider the family $\mathcal{F}^{\prime}$ constructed in Theorem 4.1. Our second claim is that $\mathcal{F}^{\prime}$ depends on at least 11 independent real parameters. In order to prove this claim, we again drop the normalization factor $1 / N$ and it suffices to show that 
the modified family

$$
\Lambda:(t, x, y, a, b, c, f, j, k, l, m, p, s) \rightarrow N \rho
$$

depends on at least 12 independent parameters.

In total we have 23 real parameters: $t, x, y$ and the real and imaginary parts of $a, b, c, f, j, k, l, m, p, s$. The domain of $\Lambda$ is an open subset of $\mathbf{R}^{3} \times \mathbf{C}^{10}$, i.e., $\mathbf{R}^{23}$. The matrix entries $\rho_{i j}$ are complex-valued rational functions of these parameters. Since the rank of the Jacobian matrix of $\Lambda$ at the point: $t=1, x=l=0, y=-1$, $c=s=\mathbf{i}, a=j=-\mathbf{i}, b=f=1-\mathbf{i}, p=1+\mathbf{i}, k=m=-1+\mathbf{i}$ is 12 , $\Lambda$ indeed depends on at least 12 independent real parameters. Hence our second claim follows.

\section{Discussion}

We have shown that the states $\rho$ of two qutrits given by Eq. (1.1) are generically entangled. The precise conditions are spelled out in Theorem 2.1. We denote by $\mathcal{F}$ the family of states $\rho$ as in Eq. (1.1) satisfying the conditions of this theorem. Although it is not obvious, one can verify that each $\rho \in \mathcal{F}$ has rank 4 , and so its eigenvalue 0 has multiplicity 5. Just as in [4, for $\rho \in \mathcal{F}$ we have $\rho_{i j}=0$ whenever $i+j$ is odd. Formally, $\mathcal{F}$ depends on 36 real parameters but only 27 of them are independent. The proof of this fact is given in section 5 .

Let us say here that a state $\rho \in \mathcal{F}$ is generic if $\rho^{\Gamma}$ is nonsingular. The family $\mathcal{F}$ contains a variety of distillable states. In Section 3 we give two such examples, $\rho_{1}$ and $\rho_{2}$, which are also generic. The former violates the reduction criterion of separability while the latter does not. We show explicitly that $\rho_{2}$ is 1-distillable. We point out that the first nontrivial example of a distillable bipartite state which satisfies the reduction criterion was given in [15].

Next we have singled out an explicit subfamily $\mathcal{F}^{\prime} \subset \mathcal{F}$ consisting of PPT-states. In fact the states $\rho \in \mathcal{F}^{\prime}$ are fixed under partial transposition, i.e., we have $\rho^{\Gamma}=\rho$ for all $\rho \in \mathcal{F}^{\prime}$. This subfamily was selected so to contain almost all members of the first Bruß-Peres family. Our family $\mathcal{F}^{\prime}$ is much richer than the latter as it depends on at least 11 independent real parameters.

Let us mention that Bruß-Peres family was used in [5] to test the effectiveness of a new separability condition. We hope that our families $\mathcal{F}$ and $\mathcal{F}^{\prime}$ will also be useful for further study of entanglement and distillability problems. There is no doubt that our first theorem admits a generalization to bipartite systems of qudits. It would be worthwhile to work out the details at least in the $4 \otimes 4$ case.

\section{REFERENCES}

[1] Baumgartner, B., Hiesmayr, B.C., and Narnhofer, H., Phys. Rev. A 74, 032327 (2006). Phys. Lett. A 372 (2008) 2190-2195;

[2] Bennett, C.H., DiVincenzo, D.P., Mor, T., Shor, P.W., Smolin, J.A. and Terhal, B.M., Phys. Rev. Lett. 82, 5385-5388 (1999).

[3] Boothby, W.M., An Introduction to Differentiable Manifolds and Riemannian Geometry, Academic Press, New York, 1975.

[4] Bruß, D., Peres, A., Phys. Rev. A 61, 030301(R) (2000).

[5] de Vincente, J.I., Quantum Inf. Comput. 7, 624 (2007).

[6] DiVincenzo, D.P., Mor, T., Shor, P.W., Smolin, J.A. and Terhal, B.M., Comm. Math. Phys. 238, 379-410 (2003).

[7] Ha, K.-C. and Kye, S.-H., J. Phys. A: Math. Gen. 38 (2005), 9039-9050.

[8] Horodecki, M. and Horodecki, P., Phys. Rev. A 594206 (1999). 
[9] Horodecki, M., Horodecki, P. and Horodecki, R., Phys. Lett. A 223 (1996) 1-8.

[10] Horodecki, M., Horodecki, P. and Horodecki, R., Phys. Rev. Lett. 80, 5239-5242 (1998).

[11] Horodecki, M., Horodecki, P. and Horodecki, R., in Quantum Information Theory: An Introduction to Basic Theoretical Concepts and Experiments, edited by G. Alber et al., Springer Tracts in Modern Physics Vol. 173 (Springer Verlag, Berlin, 2001), pp. 151-195.

[12] Horodecki, P., Phys. Lett. A 232 (1997) 333-339.

[13] Kim, W.C. and Kye, S.-H., Phys. Lett. A 369 (2007) 16-22.

[14] Peres, A., Phys. Rev. Lett. 77, 1413-1415 (1996).

[15] Shor, P.W., Smolin, J.A. and Terhal, B.M., Phys. Rev. Lett. 86, 2681-2684 (2001).

Department of Pure Mathematics, University of Waterloo, Waterloo, Ontario, N2L 3G1, CANADA

E-mail address: djokovic@uwaterloo.ca 